\title{
A Novel, Non-Cytotoxic, Anti-Invasive Therapeutic Agent for Ovarian Cancer
}

\author{
S. D. SWENSON ${ }^{\mathrm{a}}$, F. S. MARKLAND ${ }^{\mathrm{b}}$ and R. MINEA \\ ${ }^{a}$ Department of Biochemistry and Molecular Biology, and Norris Comprehensive \\ Cancer Center, Keck Medicine, University of Southern California,Los Angeles, \\ California 90089, United States \\ ${ }^{\mathrm{b}}$ Department of Neurological Surgery, Keck Medicine, University of Southern \\ California Los Angeles, California, 90089, United States \\ www.usc.edu \\ *markland@usc.edu \\ ${ }^{*}$ Corresponding author
}

Keywords: Ovarian Cancer, Integrins, Disintegrins, Intraperitoneal Delivery, Targeted Therapy.

\begin{abstract}
The 5-year survival is only about $30 \%$ when ovarian cancer (OC) is first detected at an advanced stage, which is the case for $\sim 70 \%$ of women with OC. The combination of debulking surgery and cytotoxic chemotherapy is the primary treatment modality for OC. There has been much interest in the use of intraperitoneal (IP) chemotherapy for OC and the National Cancer Institute of the USA has endorsed this form of therapy. Disintegrins represent a class of disulfide-rich polypeptides, originally isolated from snake venom, many of which contain a cyclic-Arg-Gly-Asp (c-RGD) motif. Due to their ability to bind to integrins that are important to cancer progression and dissemination, disintegrins hold a significant translational potential as anti-cancer therapeutic agents. Integrins are heterodimeric adhesion receptors on the surface of cells that relay signals bi-directionally across the plasma membrane between the extracellular matrix and cytoskeletal proteins and signaling molecules inside the cell. They are closely associated with OC progression and dissemination. Multiple studies have demonstrated that adding IP therapy to intravenous therapy produces survival benefits in patients with advanced OC. We report the use of IP delivery of a recombinant disintegrin, vicrostatin $(\mathrm{VCN})$, in mouse models of OC, which leads to highly effective inhibition of progression and dissemination of OC. When impregnated in a gel formulation VCN has extended time-release activity and retains therapeutic levels for 7-10 days; with weekly VCN injections activity is retained for prolonged times. This IP delivered formulation is effective against several human ovarian cancer cell lines, and warrants further translational studies.
\end{abstract}

\section{Introduction}

OC is a devastating disease with the serous histotype found in $~ 70 \%$ of cases and high-grade serous ovarian cancer (HGSOC) a most aggressive, highly- invasive subtype, which accounts for $90 \%$ of serous carcinomas and two-thirds of all OC deaths [1]. The standard treatment is aggressive surgery followed by adjuvant platinum-taxane chemotherapy. However, no efficient therapeutic method aimed at stopping OC dissemination is currently available in the clinic [2]. There has been much interest in the use of regional (intraperitoneal, IP) chemotherapy for OC and the National Cancer Institute of the United States has endorsed this treatment following publication of the 
results of three large randomized Phase III clinical trials [3].

Disintegrins represent a class of disulfide-rich polypeptides, originally isolated from snake venom, which hold a significant translational potential as anti-cancer therapeutic agents. Disintegrins bind only to the activated conformation of integrins in motile cells such as cancer cells and angiogenic endothelial cells. Vicrostatin (VCN) is a monomeric disintegrin of $\sim 7.1 \mathrm{kDa}$ that has been recombinantly expressed in E. coli. Its sequence is based on the natural snake venom disinte grin contortrostatin, a dimer found in the venom of the southern copperhead snake [4]. VCN has an Arg-Gly-Asp (RGD) motif at the tip of a flexible loop stabilized by disulfide bonds [4]. Interaction of VCN with multiple integrins (i.e., $\alpha v \beta 3, \alpha v \beta 5, \alpha 5 \beta 1$ and $\alpha \mathrm{IIb} \beta 3$ ), primarily through the RGD motif [4], enables it to target both endothelial and OC cells. Potent antitumor activity of VCN has been previously described in models of both breast and prostate cancer $[4,5]$. There were no visible side effects or signs of internal bleeding, indicating that in mice chronic administration of VCN is not cytotoxic [4].

Integrins are heterodimeric adhesion receptors on the cell surface that relay signals bi-directionally across the plasma membrane between the extracellular matrix and signaling pathways and cytoskeletal proteins [6]. The $\square$ subunits combine with $\square$ subunits to create 24 unique $\square \square$ heterodimers resulting in a range of specificities toward extracellular matrix (ECM) proteins [6]. Several members of the integrin family recognize the RGD sequence present in ECM proteins [7]. Integrins (including $\alpha v \beta 3$, $\alpha v \beta 5, \alpha 5 \beta 1$ ) have important roles in OC cell attachment, survival, migration, invasion and angiogenesis [8], and their involvement in processes critical to tumor progression suggests they would serve as attractive targets for cancer therapy [9]. The broad spectrum of integrin targets to which VCN binds make it an attractive therapeutic agent.

\section{Materials and Methods}

\section{Materials}

Vicrostatin (VCN) was recombinantly produced and HPLC purified according to an established protocol [4]. Oxiplex gel was obtained from FizioMed, San Luis Obispo, CA. OVCAR3 and SKOV3 cells were obtained from ATCC (Bethesda, MD). Both cell lines have been stably transfected by an adenovirus containing genes coding for luciferase (luc) and green fluorescent protein (GFP).

\section{Cell Line and Culture}

The established human ovarian cancer cell lines OVCAR3 and SKOV3 were used in these studies and were grown as monolayers in vitro. Cells were grown in RPMI 1640 (OVCAR-3), and McCoy's 5a modified medium (SKOV-3), at $37^{\circ} \mathrm{C}$ in a humidified incubator (5\% CO2 and 95\% air). Cells were harvested with trypsin-EDTA, washed with complete medium, and resuspended in PBS.

Spheroids are multicellular aggregates that can be grown in vitro from any human OC cell line by seeding cells in appropriate media and forcing the cells to grow in suspension by plating them on low binding surfaces. To generate spheroids from

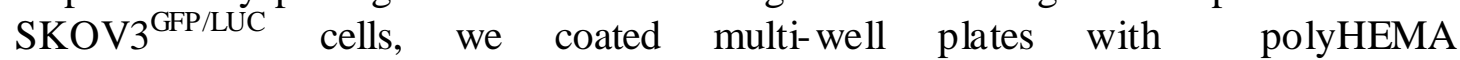
(poly\{2-hydroxyethyl methacrylate\}), and seeded cells in their regular medium and allowed them to form spheroids. Briefly, $1 \times 10^{6}$ cells were seeded per well (in 6-well plates) and allowed to aggregate for at least 48 hours. Then the spheroids were resuspended at a density of $20 \times 10^{6}$ cell equivalents $/ \mathrm{ml}$. 


\section{Animal Tumor Model}

For the dispersed cell model both OVCAR3 and SKOV3 cells growing as a monolayer in culture were harvested and cells $\left(2 \times 10^{7}\right)$ were suspended in $1 \mathrm{ml}$ PBS and $100 \mu \mathrm{lof}$ the cell suspension was injected IP into five-week old female athymic nude mice (Balb/c/nu/nu). Each animal received $2 \times 10^{6}$ cells, dispersed cells or cell equivalents in the spheroid models, and the tumors were allowed to implant for 4-14 days before treatment was initiated; tumor growth was confirmed by bioluminescent imaging.

\section{Release Kinetics of VCN from Oxiplex}

We evaluated release kinetics for a number of different formulations of VCNimpregnated Oxiplex gel. We used identical volumes of Oxiplex $(1 \mathrm{ml})$ impregnated with 1,3 or $10 \mathrm{mg} / \mathrm{ml} \mathrm{VCN}$, and determined release kinetics.

\section{Animal Imaging}

To evaluate presence of tumor prior to initiating treatment and biweekly throughout the study, mice were imaged at the Molecular Imaging Center Core. Mice were injected intravenously with $50 \mathrm{mg} / \mathrm{kg}$ of D-luciferin in PBS 90 seconds prior to imaging. Imaging was performed using a charge-coupled device camera (IVIS 200, Xenogen-Caliper, Alameda, CA). Mice were anesthetized with isoflurane prior to and during imaging. Total photon flux (photons/sec) was measured from a fixed region-of-interest over the peritoneal space of the mice using Living Image software (Perkin Elmer, Waltham MA) [10].

\section{Results}

\section{Release Kinetics of VCN from Oxiplex}

Oxiplex (1 ml) impregnated with 1,3 or $10 \mathrm{mg} / \mathrm{ml} \mathrm{VCN}$ was evaluated and it was determined that the release kinetics varied inversely with drug concentration (i.e., $10 \mathrm{mg} / \mathrm{ml}$ being the slowest). For these studies, a very small volume of VCN ( 50ul) was mixed with $1 \mathrm{ml}$ of Oxiplex, ensuring that the gel was not diluted by more than $5 \%$. VCN incorporated into Oxiplex at $2-10 \mathrm{mg} / \mathrm{ml}$ resulted in a sustained release of the drug that lasted for 10-12 days (Figure 1).

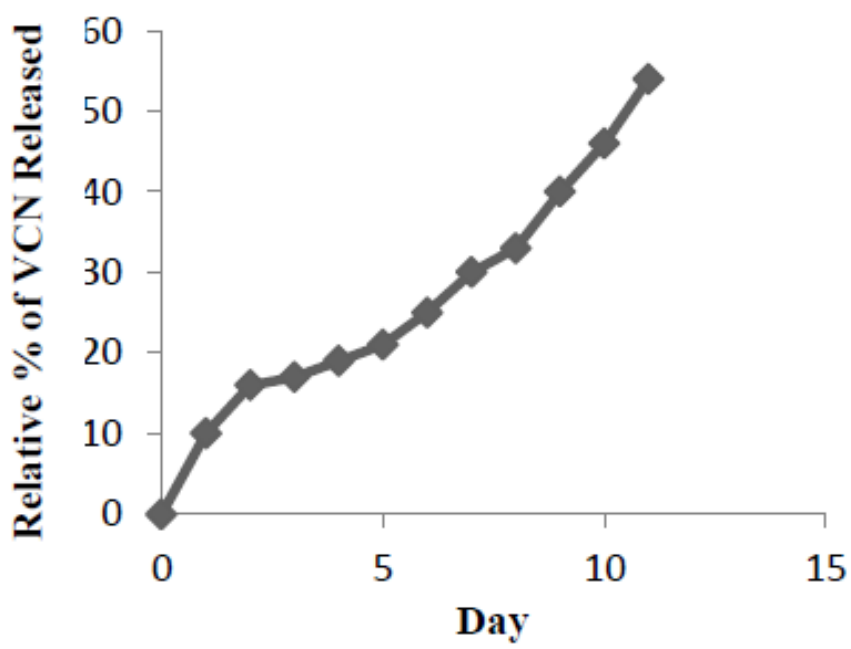

Figure 1. Release of VCN from Oxiplex. 
This sample was evaluated by measurement of released soluble $125 \mathrm{I}-\mathrm{VCN}$. VCN when impregnated in Oxiplex gel is released with an initial rapid rate of $\sim 16 \%$ of the loaded material over the initial 2 days. The gel remains intact for $>11$ days releasing nearly $55 \%$ of the loaded VCN. Retention of VCN Efficacy

Preliminary assess- ment of VCN- Oxiplex in a murine model of human OC was performed. OVCAR 3 cells $\left(2 \times 10^{6}\right)$ were injected IP in nude mice and two weeks were allowed for tumors to develop before $\mathrm{VCN}$ - Oxiplex therapy was administered for four weeks. The animals received weekly either $1 \mathrm{ml}$ Oxiplex alone or $1 \mathrm{ml}$ Oxiplex impre gnated with VCN $(10 \mathrm{mg} / \mathrm{ml})$. At the end of the study the animals were dissected; the control group showed widespread tumor throughout the peritoneal cavity, while VCN-treated animals were devoid of macroscopic tumors on visual inspection.

\section{Dose Response/Frequency Studies of VCN-Oxiplex}

A dose-response study with VCN-Oxiplex using SKOV3 human OC cells was performed. These studies evaluated the effect on tumor dissemination of the administration of 1, 2.5 and $5 \mathrm{mg} \mathrm{VCN}$ in $1 \mathrm{ml}$ Oxiplex delivered once per week, vers us Oxiplex alone. Treated groups showed a significant decrease in the number of macroscopic foci as the VCN concentration increased; animals that received $5 \mathrm{mg} / \mathrm{ml}$ of VCN-Oxiplex weekly showed no visible tumor.

To demonstrate the reliability of the bioluminescent signal from the luciferase expressing tumors, the SKOV3 ${ }^{\text {GFP/LUC }}$ model was utilized and an inoculum of $2 \times 10^{6}$ cells was injected IP and tumors allowed to grow for 2 weeks prior to the initiation of therapy. Therapy consisted of $5 \mathrm{mg}$ VCN delivered in $1 \mathrm{ml}$ Oxiplex/week versus $1 \mathrm{ml}$ Oxiplex alone/week for a 4-week course. VCN treated animals were essentially devoid of visible macroscopic tumor foci by gross examination, while Oxiplex alone animals showed extensive tumor dissemination throughout the peritoneal cavity. Bioluminescent imaging quantitation showed greater than $95 \%$ inhibition of tumor spread in the treated group after 4 weeks of treatment.

\section{VCN-Oxiplex VS. VCN-Saline}

In this study animals (10 animals per group) were inoculated with $0.1 \mathrm{ml}$ of SKOV3 ${ }^{\text {GEPLUC }}$ spheroids ( $2 \times 10^{6}$ cell equivalent) IP and the spheroids were allowed to implant for 4 days. The animals received IP weekly $1 \mathrm{ml}$ doses of either $5 \mathrm{mg}$ of VCN in saline, $5 \mathrm{mg}$ of VCN in Oxiplex, or Oxiplex alone. Animals in the Oxiplex alone group showed extensive carcinomatosis that was disseminated throughout the peritoneal cavity. Animals that received VCN- saline or VCN-Oxiplex showed dramatically less or no visible macroscopic tumor foci by gross examination (Fig. 2). Quantitation of bioluminescent imaging reveals that VCN-Oxiplex and VCN-saline inhibit tumor growth by $\sim 95-98 \%$ (Figure 3 ). These results indicate that Oxiplex and saline delivery modalities are similarly effective. 


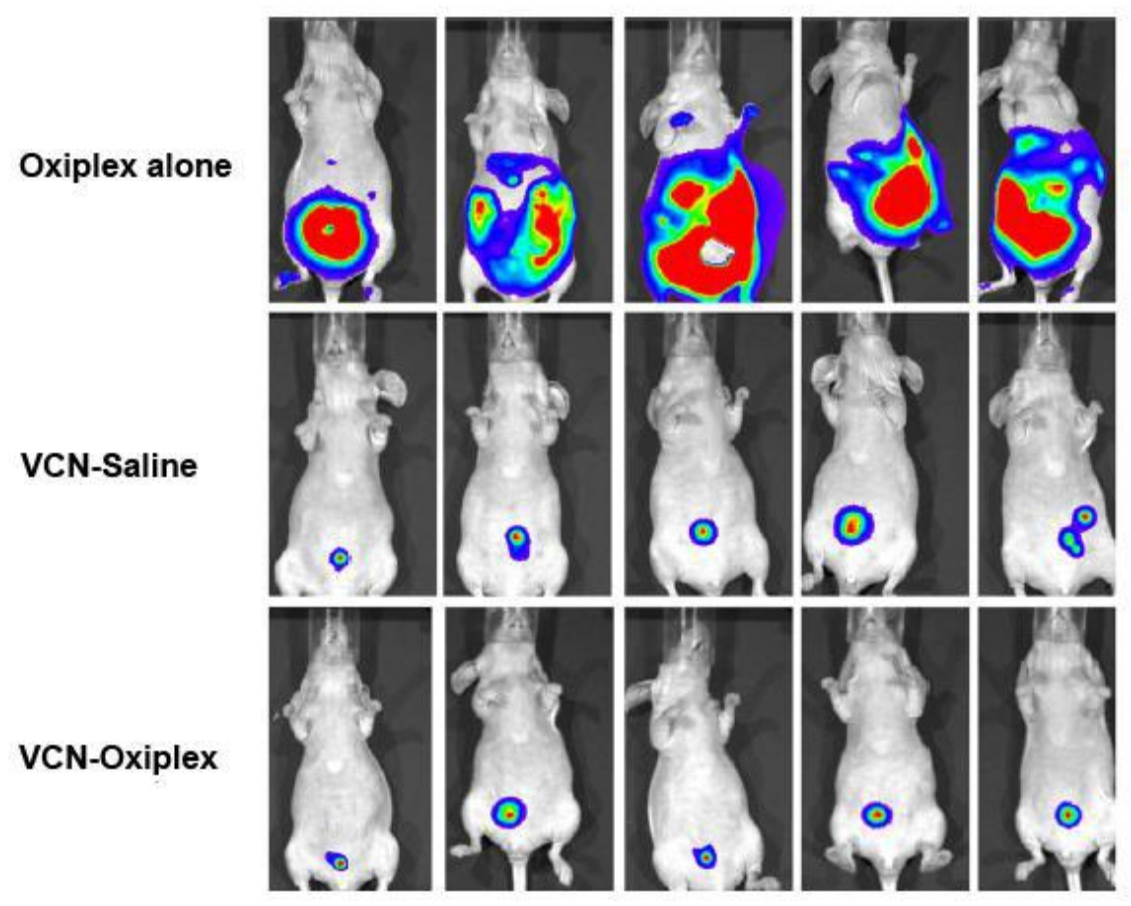

Figure 2. Bioluminescent Images of Representative Mice after 4 Weeks of Treatment with either VCN-Saline or VCN-Oxiplex versus Control. Representative mice from groups of 10 mice are shown after 4 weeks of treatment. Beginning 4 days post spheroid implantation, mice were treated via IP injection with VCN either in saline or Oxiplex.

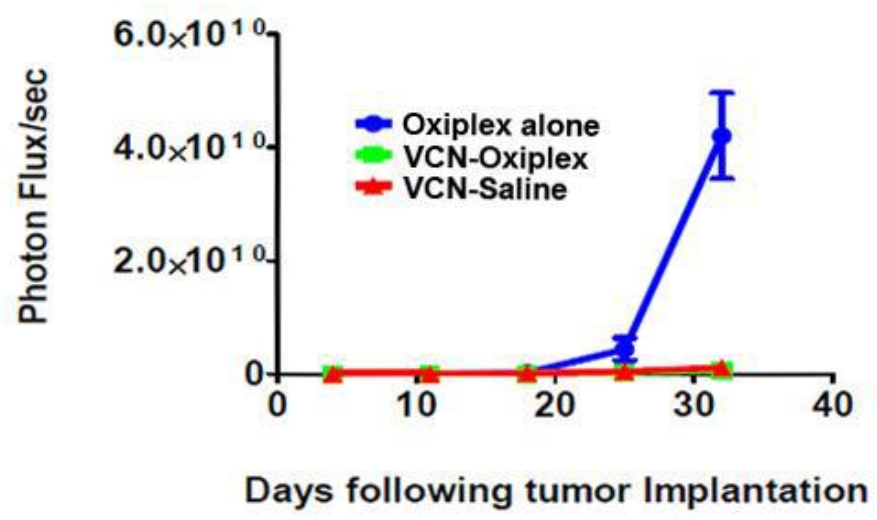

Figure 3. Bioluminescent Quantitation of Tumors. Mice in the SKOV3GFP/LUC spheroid model were imaged weekly beginning on day 4 post tumor implantation. The animals were anesthetized and the luciferin substrate $(50 \mathrm{mg} / \mathrm{kg}$ ) was injected IV. Sixty second s following luciferin injection, mice were placed in the Xenogen Ivis 200 optical imaging unit and images were acquired for 1 and 60 seconds. For quantitation, the photon flux of bioluminescent areas was averaged and plotted over time for each group.

\section{Discussion}

Consistent findings in HGSOC include the activation of multiple components of the cellular invasion apparatus (i.e., invadosome-associated integrins and their downstream kinases) [11]. A more efficient and less toxic form of therapy aimed at addressing metastatic residual OC following surgery is urgently needed to prolong the survival and 
improve the quality of life of these patients. OC spheroids, unlike individual OC cells, are known to be much more resistant to chemotherapy. Unlike primary tumors and macrometastases, OC micrometastases evade detection at the time of surgery, and failure to eradicate them is the main reason for recurrence. We believe that an effective way of addressing this clinical problem is by the means of a broad-spectrum antiinvasive agent, and VCN represents such an anti-invasive therapeutic.

What complicates the management of OC is that chemotherapy administered intravenously (IV) penetrates only poorly into the peritoneal compartment where the disease is actually spreading. One solution to this problem is the administration of chemotherapy directly to the intraperitoneal space after debulking surgery, and in advanced OC a $21.6 \%$ decrease in the risk of death was reported in patients undergoing combined IP and IV therapy versus those undergoing IV therapy alone [12]. Although efficacious, the regimens of currently used IP platinum and taxane agents are accompanied by dramatic off- target toxic effects. This is the main reason why a significant number of women opt out of the IP treatment despite its potential benefits.

We propose a radically new approach aimed at delaying the further dissemination of $\mathrm{OC}$ in the post-debulking surgery setting, and we have developed a non-toxic engineered disintegrin, a peptide called vicrostatin (VCN) with broad-spectrum anti-integrin activity (i.e., against multiple $\square \mathrm{v}$ and 5 integrin members that are uniformly upregulated in $\mathrm{OC}$ ). $\mathrm{VCN}$ acts through a mechanism that involves high-specificity binding to invadosome-associated tumoral integrins displayed by OC cells [13]. This anti-invasive mechanism is experimentally supported by encouraging preliminary studies with xenograft models of OC, as described here. At the molecular level VCN demonstrates its anti-invasive activity by disrupting the actin cytoskeleton organization as we previously showed [4]. To our knowledge, a therapeutic solution like this, involving delivery of a targeted, anti-invasive drug directly in the IP space in OC patients immediately after debulking surgery has never been tested in clinic.

\section{Acknowledge ments}

The content is solely the responsibility of the authors and does not necessarily represent the official views of the National Institutes of Health.

Competing Interests: Drs. Markland, Minea and Swenson are co-founders of Disintegrin Therapeutics, Inc., a start-up company aimed at developing disintegrin-based therapeutic and diagnostic solutions.

This work was partially supported by the National Cancer Institute, National Institutes of Health of the United States: Award number 1R41 CA168228.

\section{References}

[1]Howlader N NA, Krapcho M, Garshell J, Miller D, Altekruse SF, Kosary CL, Yu M, Ruhl J, Tatalovich Z,Mariotto A, Lewis DR, Chen HS, Feuer EJ, Cronin KA, editor. SEER Cancer Statistics Review, 1975-2012. Bethesda, MD: National Cancer Institute, 2015.

[2]American Cancer Society Cancer Facts and Figures 2016. Atlanta, GA 2016.

[3] Trimble EL, Alvarez RD. Intraperitoneal chemotherapy and the NCI clinical announcement. Gynecol Oncol 2006;103(2 Suppl 1):S18-9.

[4]Minea RO, Helchowski CM, Zidovetzki SJ, Costa FK, Swenson SD, Markland FS, Jr. Vicrostatin - an anti-invasive multi-integrin targeting chimeric disintegrin with 
tumor anti-angiogenic and pro-apoptotic activities. PloS one 2010;5(6):e10929.

[4]Lin E, Wang Q, Swenson S, Jadvar H, Groshen S, Ye W, et al. The disintegrin contortrostatin in combination with docetaxel is a potent inhibitor of prostate cancer in vitro and in vivo. Prostate 2010;70(12):1359-70.

[5]Askari JA, Buckley PA, Mould AP, Humphries MJ. Linking integrin conformation to function. J Cell Sci 2009;122(Pt 2):165-70.

[6]Ruoslahti E. Integrins. The Journal of clinical investigation 1991;87(1):1-5.

[7]Hapke S, Kessler H, Luber B, Benge A, Hutzler P, Hofler H, et al. Ovarian cancer cell proliferation and motility is induced by engagement of integrin alpha(v)beta3/Vitronectin interaction. Biol Chem 2003;384(7):1073-83.

[8]Desgrosellier JS, Cheresh DA. Integrins in cancer: biological implications and therapeutic opportunities. Nat Rev Cancer 2010;10(1):9-22.

[9]Gross S, Piwnica-Worms D. Monitoring proteasome activity in cellulo and in living animals by bioluminescent imaging: technical considerations for design and use of genetically encoded reporters. Methods Enzymol 2005;399:512-30.

[10]Lengyel E. Ovarian cancer development and metastasis. Am J Pathol 2010;177(3):1053-64.

[11]Jaaback K, Johnson N, Lawrie TA. Intraperitoneal chemotherapy for the initial management of primary epithelial ovarian cancer. Cochrane Database Syst Rev 2011(11):CD0053.

[12]Davidson B, Goldberg I, Reich R, Tell L, Dong HP, Trope CG, et al. AlphaVand beta1-integrin subunits are commonly expressed in malignant effusions from ovarian carcinoma patients. Gynecologic oncology 2003; 90(2):248-57. 